\title{
INTENSIDADE ECOLÓGICA DO BEM-ESTAR NO BRASIL: UMA ABORDAGEM REGIONAL
}

Ely José de Mattos 1 Camila Horst Toigo ${ }^{2}$ Osmar Tomaz de Souza ${ }^{3}$

Resumo: A literatura moderna sobre a relação entre atividade humana e pressão ambiental tem crescido. Uma das abordagens recentes é relacionar o crescimento econômico com a intensidade ecológica do bem-estar humano (EIWB), que é medida através da razão de uma variável de pressão ambiental e uma variável de bem-estar humano. O presente artigo investiga os efeitos do crescimento econômico sobre a EIWB no Brasil e suas grandes regiões entre 2002 e 2014. Para isso, utilizou-se o modelo de regressão time-series cross-section (TSCS) Prais-Winsten. Os resultados apontam que o crescimento econômico está associado ao aumento da intensidade ecológica do bem-estar para o Brasil como um todo para o período analisado. Quando observados os resultados para as regiões, identifica-se que as regiões Norte, Nordeste e Centro-Oeste replicam este efeito. As regiões Sul e Sudeste não confirmam tal resultado.

Palavras-chave: Intensidade ecológica do bem-estar humano. Crescimento econômico. Sustentabilidade.

\section{ECOLOGICAL INTENSITY OF WELL-BEING IN BRAZIL: A REGIONAL APPROACH}

Abstract: The literature on the relationship between the human activity and environmental pressure has been expanding. One of the most recent approaches is the one which stablishes a relationship between economic growth and the ecological intensity of human well-being (EIWB). The EIWB is measured through the ratio between a variable of environmental pressure and a variable of well-being. This article investigates the effects of economic growths in Brasil and its regions in the 2002-2014 period. In order to achieve that, it has been used a Prais-Winsten Timeseries cross-section (TSCS) regression model. The results point out that que economic growth is associated with an increase in the EIWB in Brazil as a whole for the period in question. As for the regional results, it has been found that North, Northeast and Centre-West regions replicate the same effect. South and Southeast regions do not confirm such a result.

Keywords: Ecological intensity of human well-being. Economic growth. Sustainability.

\footnotetext{
${ }_{1}^{1}$ Pontifícia Universidade Católica do Rio Grande do Sul, Curso de Economia, Porto Alegre, Brasil, ely.mattos@pucrs.br, https://orcid.org/0000-0002-6364-1599

2 Doutora em Economia pela Pontifícia Universidade Católica do Rio Grande do Sul, Porto Alegre, Brasil, camilahorstoigo@gmail.com, https://orcid.org/0000-0002-1491-2508

${ }^{3}$ Pontifícia Universidade Católica do Rio Grande do Sul, Curso de Economia, Porto Alegre, Brasil, osmar.souza@pucrs.br, https://orcid.org/0000-0002-9854-8642
} 


\section{INTENSIDAD ECOLÓGICA DEL BIENESTAR EN BRASIL: UN ENFOQUE REGIONAL}

Resumen: La moderna literatura sobre la relación entre actividad humana y presión ambiental ha crecido. Uno de los enfoques recientes es relacionar el crecimiento económico con la intensidad ecológica del bienestar humano (EIWB), que se mide via la razón de una variable de presión ambiental y una variable de bienestar humano. El presente artículo investiga los efectos del crecimiento económico sobre la EIWB en Brasil y sus regiones entre 2002 y 2014. Para tal, se utilizó el modelo de regresión time-series cross-section (TSCS) Prais-Winsten. Los resultados apuntan que el crecimiento económico está asociado al aumento de la intensidad ecológica del bienestar para Brasil como un todo para el período analizado. Cuando se observan los resultados para las regiones, se identifica que las regiones Norte, Nordeste y Centro-Oeste replican este efecto. Las regiones Sur y Sudeste no confirman tal resultado.

Palabras clave: Intensidad ecológica del bienestar humano. Crecimiento econômico. Sostenabilidad.

\section{Introdução}

O debate a respeito do desenvolvimento sustentável, que tomou forma na segunda metade do século XX e intensificou-se após a Rio+20, trouxe uma visão sistêmica sobre os diversos desequilíbrios que se estabelecem na relação entre sociedade e meio ambiente. Trata-se de um tema em constante evolução, sobretudo porque ainda existem divergências e ambiguidades a respeito do que é ser/estar sustentável, do que é ser/estar desenvolvido e sobre as conexões existentes entre desenvolvimento e meio ambiente no longo prazo (ROMEIRO, 1999; FOLADORI, 2001; PARRIS e KATES, 2003).

Há décadas, vasta literatura vem destacando que os padrões vigentes de consumo, de produção e de desenvolvimento são geradores de degradação ambiental e de mudança climática (MEADOWS, et al. 1972; DALY, 1984; WACKERNAGEL e REES, 1996; JACKSON, 2009; STEINBERGER, et al. 2012). Trabalhos diversos já apontaram haver correspondência direta entre variáveis socioeconômicas e emissões de gases do efeito estufa e pegada ecológica, como Ravallion et al. (2000), Jorgenson (2014); Grunewald et al. (2017), Fan et al., (2006), Dietz e Rosa (1994), Knight (2014), salientando que as ações antropogênicas têm sido responsáveis, ao menos em parte, pelos níveis atuais de $\mathrm{CO} 2$ emitidos, o que vai ao encontro dos últimos relatórios divulgados pelo Intergovernmental Panel on Climate Change (IPCC, 2014). 
O interesse em analisar a relação entre degradação ambiental e condições socioeconômicas popularizou-se com o trabalho de Grossman e Krueguer (1991), que propunha a ideia da existência de uma associação não linear, em forma de $U$ invertido, entre crescimento econômico e pressão ambiental. A Curva Ambiental de Kuznets (CAK), como ficou conhecida, reza que nas fases iniciais do crescimento econômico, a pressão ambiental aumenta para depois, quanto atingido determinado patamar, o crescimento passar a estar associado à redução do impacto ambiental.

Então, a partir de meados da década de 1990, a CAK passou a ser importante referência metodológica e conceitual para o estudo das relações entre pressão ambiental e crescimento econômico (ARRAES et al., 2006; DINDA, 2004). Com a intensificação dos debates sobre os impactos antropogênicos nos ambientes naturais, catalisados sempre por novas evidências, o escopo analítico evoluiu em volume e complexidade, tornando-se necessário um aperfeiçoamento do próprio conceito de sustentabilidade enquanto constructo que envolve não apenas a dimensão ambiental, mas também a econômica e social (MA, 2003).

Em um primeiro momento, há o reconhecimento de que a busca por elevar o bem-estar humano a partir do crescimento econômico acentua a dependência dos recursos e serviços ecossistêmicos, aprofundando a indução antrópica de impactos ambientais (JORGENSON e DIETZ, 2015; TEEB, 2001; MA, 2005). Para construir um caminho mais equilibrado no que diz respeito à relação sociedade-natureza, a geração de bem-estar humano deve ser compatível com os limites naturais (JACKSON, 2009).

O bem-estar está relacionado à eficiência do desenvolvimento econômico no sentido da ampliação dos benefícios que ele promove à vida das pessoas, que é a tese central da abordagem do desenvolvimento humano consolidada na década de 1990 (UNDP, 1990, SEN, 2001). Quando se consideram os aspectos ambientais envolvidos, o conceito de desenvolvimento sustentável tende a ponderar os resultados do processo de ampliação do bem-estar (MA, 2003). O próprio surgimento da Economia Ecológica se dá em um contexto que aponta o meio ambiente não como uma barreira ou mero fornecedor de insumos, mas como elemento intrinsicamente ligado a dinâmica econômica e ao bem-estar das pessoas (DALY e FARLEY, 2004).

O refinamento desse debate apontou para a elaboração de métricas e abordagens distintas, que visam investigar a pressão exercida sobre o meio ambiente para viabilizar a geração de bem-estar humano. Dietz, et al. (2012), então, 
com a intenção de testar a existência da CAK de forma alternativa, propuseram a definição de uma nova medida: a intensidade ecológica do bem-estar humano (ecological intensity of human well-being, EIWB, no original), que passou a representar a quantidade de estresse depositado no meio ambiente na geração de uma unidade de bem-estar (DIETZ et al., 2012). A EIWB é compreendida e empiricamente mensurada a partir da relação entre uma variável não monetária representativa do bem-estar e uma medida de estresse ambiental. Dietz et al. (2012) utilizaram a razão da Pegada Ecológica per capita com a expectativa de vida como a variável EIWB. O seu trabalho tratou de avaliar a relação entre esta medida, a EIWB, e o crescimento econômico. Como resultado, identificou uma relação em formato de U, que é o inverso da CAK tradicional. Foi o primeiro estudo neste sentido, abrindo novas possiblidades de análise.

Jorgenson e Dietz (2015) também utilizaram como medida de EIWB a relação entre a Pegada Ecológica per capita e a expectativa de vida ao nascer. Eles identificaram que desde os anos 1970, os países mais desenvolvidos têm demonstrado aumento na EIWB conforme ocorre crescimento econômico, ou seja, maior pressão ambiental para geração de bem-estar. Quando restrito apenas à emissão de CO2, ao invés da Pegada Ecológica, Jorgenson (2014) alerta que a intensidade de emissão de carbono deverá crescer junto com a evolução de bemestar humano no futuro.

No trabalho de Jorgenson e Givens (2011) foi utilizada a relação entre as emissões de carbono em função do consumo e a expectativa de vida como variável dependente. Os resultados da pesquisa apontam na direção de que, ao longo do tempo, se consolidou um aumento do impacto do crescimento econômico sobre esta variável, de modo especialmente marcado para os países em desenvolvimento.

No Brasil, em particular, o debate sobre a relação entre desenvolvimento e meio ambiente tem sido calcado em avaliações mais tradicionais, que envolvem análises custo-benefício e valoração ambiental, curva ambiental de Kuznets e indicadores de sustentabilidade compostos. Contudo, a ideia da EIWB ainda não é explorada. Pesquisas como as de Carvalho e Almeida (2010) e Arraes, et al. (2006) buscaram relacionar desenvolvimento econômico e emissão de dióxido de carbono a partir da verificação da ocorrência da CAK; outros trabalhos, como o de Mattos e Fillipi (2014), investigaram os potenciais indutores de impactos ambientais intrínsecos ao processo de desenvolvimento, contudo, pautaram-se em aspectos econômicos e demográficos. Nenhum destes estudos, no entanto, avançou na 
relação entre crescimento econômico, impacto ambiental e bem-estar, enquanto dimensões complexas e interligadas.

Portanto, no intuito de avançar nesse debate, considerando especificamente $o$ contexto brasileiro, este trabalho propõe investigar os efeitos do crescimento econômico sobre a intensidade ecológica do bem-estar humano (EIWB) no país e suas grandes regiões entre 2002 e 2014, considerando informações referentes à 26 unidades federativas. Para isso, utilizou-se o modelo de regressão Time-series Cross-section Prais-Winsten (TSCS) com erros-padrão corrigidos para painel. Como medida de estresse ambiental propomos as emissões per capita de dióxido de carbono (CO2) em toneladas e, como representantes do bem-estar humano, a esperança de vida ao nascer e a proporção de pessoas em situação de pobreza monetária, conforme sugere a literatura já existente sobre o tema. A próxima seção traz o detalhamento dos dados e método.

\section{Dados e método}

O objetivo do trabalho é testar empiricamente o impacto do crescimento econômico sobre a intensidade ecológica do bem-estar, representada pela variável EIWB. Foi montado um painel para o período de 2002 a 2014, considerando 26 Estados brasileiros ${ }^{4}$, totalizando 338 observações. A estratégia principal é estimar estes impactos para o Brasil como um todo e também para cada uma das grandes regiões, para avaliar se os efeitos podem ser generalizados ou guardam particularidades regionais. As variáveis utilizadas no trabalho estão listadas na Tabela 01.

Tabela 01 - Variáveis utilizadas no trabalho e suas fontes

\begin{tabular}{l|c}
\hline \multicolumn{1}{c|}{ Variável } & Fonte \\
\hline Produto Interno Bruto & Conta Regionais IBGE \\
\hline População & $\begin{array}{c}\text { Projeções populacionais } \\
\text { IBGE }\end{array}$ \\
\hline Expectativa de vida ao nascer & $\begin{array}{c}\text { Indicadores de } \\
\text { Desenvolvimento Sustentável } \\
\text { do IBGE }\end{array}$ \\
\hline Pobreza (\% população) & IPEA \\
\hline Emissões de CO2 & $\begin{array}{c}\text { Sistema de Estimativa de } \\
\text { Emissões de Gases Efeito } \\
\text { \% do PIB em agropecuária }\end{array}$ \\
\hline$\%$ do PIB em serviços & Conta Regionais IBGE \\
\hline$\%$ do PIB em indústria & Conta Regionais IBGE \\
\hline$\%$ da população ativa & Conta Regionais IBGE \\
\hline
\end{tabular}

${ }^{4} \mathrm{O}$ Distrito Federal, por suas características socioeconômicas e políticas peculiares, o que faz com que seja considerado um outlier, foi a única UF retirada da base de dados. 


\begin{tabular}{l|l}
\hline & IBGE \\
\hline Coeficiente de Gini da renda & IPEA \\
\hline
\end{tabular}

Fonte: elaborado pelos autores.

A variável EIWB foi construída conforme sugerido por Dietz et al. (2012) e Jorgenson e Dietz (2015). Para representar o estresse ambiental gerado por causas antrópicas utilizamos as emissões de $\mathrm{CO} 2$ per capita, medido em toneladas, disponibilizadas pelo Sistema de Estimativa de Emissões de Gases Efeito Estufa. Mais especificamente, utilizamos o CO2 equivalente GWP AR5. A escolha deste gás justifica-se pelo fato de que dentre os gases mais importantes para a geração do efeito estufa, o dióxido de carbono é um dos principais gases emitidos em decorrência das atividades humanas (IPCC, 2014).

A representação do bem-estar humano foi determinada a partir de duas variáveis: a expectativa de vida ao nascer, que indica quantos anos, em média, uma criança viveria se mantido o padrão de mortalidade do período analisado, e o percentual de pessoas pobres com renda domiciliar per capita inferior à linha de pobreza. A escolha da primeira variável teve respaldo nas justificativas apresentadas na pesquisa de Dietz, et al. (2012), que a aponta como um indicador fortemente aceito como representante de bem-estar social, além de ser mensurada em muitos países e, geralmente, calculada por sistemas nacionais de dados demográficos, o que eleva a qualidade e a validade do dado.

Já a escolha da segunda variável justifica-se pelo fato de que os níveis altos de pobreza no Brasil ainda configuram importante característica a ser levada em conta quando se avalia a média da qualidade de vida brasileira. Apesar dos avanços já visíveis na redução da desigualdade de renda e no combate à pobreza (IPEA, 2019), o Brasil ainda concentra um número importante de pessoas vulneráveis social e economicamente, o que reflete fragilidade e ineficiência na geração de bem-estar humano.

Assim, a EIWB foi calculada a partir das relações esboçadas nas equações (1) e (2), onde as emissões são apresentadas em toneladas per capita, a expectativa de vida em anos e a pobreza em percentual da população.

$$
E I W B_{1}=\frac{\mathrm{CO}_{2} \text { per capita }}{\text { expectativa de vida }}
$$


$E I W B_{2}=\frac{\mathrm{CO}_{2} \text { per capita }}{\text { proporção de indivíduos pobres }}$

A variável escolhida para representar o desenvolvimento econômico dos 26 estados foi o Produto Interno Bruto per capita, calculado a partir da relação entre os PIB estaduais e as projeções populacionais. Destaca-se que os valores divulgados para o PIB foram dispostos em termos nominais, portanto, foi necessário deflacionálos pelo Índice Geral de Preço - Disponibilidade Interna (IGP-DI) aos preços de 2014.

Além destas, foram incluídas algumas variáveis de controle. O percentual do PIB por setor, a porção de população ativa e também o coeficiente de Gini são variáveis que avaliamos como heterogêneas e dinâmicas entre os estados ao longo do tempo, de modo que não seriam plenamente absorvidas com efeitos fixos.

O modelo econométrico utilizado exigiu que a base de dados estivesse balanceada, isto é, que cada indivíduo em corte transversal (no caso desta pesquisa, os estados) obtivesse o mesmo número de observações. Para tanto, fezse uma interpolação linear para o ano de 2010 para as variáveis proporção de indivíduos pobres e coeficiente de Gini, que não dispunham de informações para esse período.

\section{Modelo econométrico}

Para verificar os efeitos do desenvolvimento econômico sobre a intensidade ecológica do bem-estar humano ao longo do tempo, utilizou-se o modelo de regressão time-series cross-section Prais-Winsten (TSCS) com erros-padrão corrigido para painel (panel-corrected standard erros - PCSE), conforme proposto em Jorgenson e Dietz (2015). Este modelo trata de perturbações heteroscedásticas e correlacionadas no painel. Diferentemente do modelo tradicional de regressão com dados em painel, um modelo TSCS é adequado em situações com um número relativamente pequeno de indivíduos observadas ao longo de um período de tempo mais longo. O modelo é corrigido pelo processo autorregressivo de primeira ordem (AR(1)) para painel, conforme as justificativa expostas por Beck e Katz (1995), que a atribui como uma característica tradicionais dos painéis.

O modelo econométrico elaborado está disposto na equação (3). 


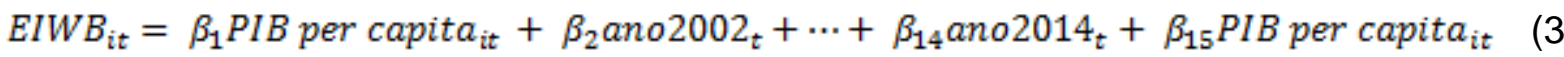

*ano2002 $+\cdots+\beta_{27}$ PIB per capita ${ }_{i t} *$ ano $2014_{t}+u_{t}+e_{i t}$

onde EIWB é a variável dependente para o estado $i$ e ano $t ; \beta$ são os coeficientes estimados; PIB per capita it $_{\text {é }}$ O PIB per capita para o estado $i$ e ano $t$, $\left(\beta_{2}\right.$ ano $2003_{t}+\cdots+\beta_{14}$ ano $\left.2014_{t}\right)$ representam variáveis dummy para cada ano; $\left(\beta_{15}\right.$ PIB per capita it $*{\text { ano } 2003_{t}}_{t}+\cdots+\beta_{27}$ PIB per capita $\left.{ }_{t} *{\left.\text { ano } 2014_{t}\right)}\right)$ são as interações entre o PIB per capita e as variáveis dummy de tempo (com 2002 como o ano de referência) para cada um dos 26 estados em cada um dos anos; $e_{i t}$ é o termo de erro de cada estado em cada ponto do tempo; e, o termo $u_{t}$ é o efeito não observado invariante no tempo de cada estado. Isto é, são os efeitos fixos para Estado, gerados sobre a variável dependente que diferem entre os estados, mas, permanecem inalterados para cada um ao longo do tempo. Portanto, o modelo funciona como uma abordagem de painel com efeitos fixos nos dois sentidos, tempo e unidade de análise.

Para a estimação, todas as variáveis sofreram transformação logarítmica, de modo que podem ser interpretados como elasticidades. Lembrando que o efeito do PIB para cada ano da série temporal analisada é obtido pela soma do coeficiente do PIB per capita e da interação [PIB per capita * ano], desde que os coeficientes sejam significativos - caso algum não seja, é considerado como zero no cálculo do efeito líquido.

\section{Resultados}

Ao longo do período analisado, o padrão de emissões no Brasil mudou consideravelmente. Houve redução significativa nas emissões de $\mathrm{CO} 2$ para maior parte dos Estados na primeira década do século XXI. A Figura 01 mostra a evolução das emissões por região do Brasil - obtidas através da soma dos volumes emitidos por cada região, dividido pela respectiva população. Atente que o gráfico utiliza 0 eixo secundário para representar as regiões Norte e Centro-Oeste, pois estas regiões têm níveis de emissão consideravelmente mais altos que as demais. 
Figura 01 - Emissões $\mathrm{CO}_{2}$ por região do Brasil

(toneladas anuais per capita)

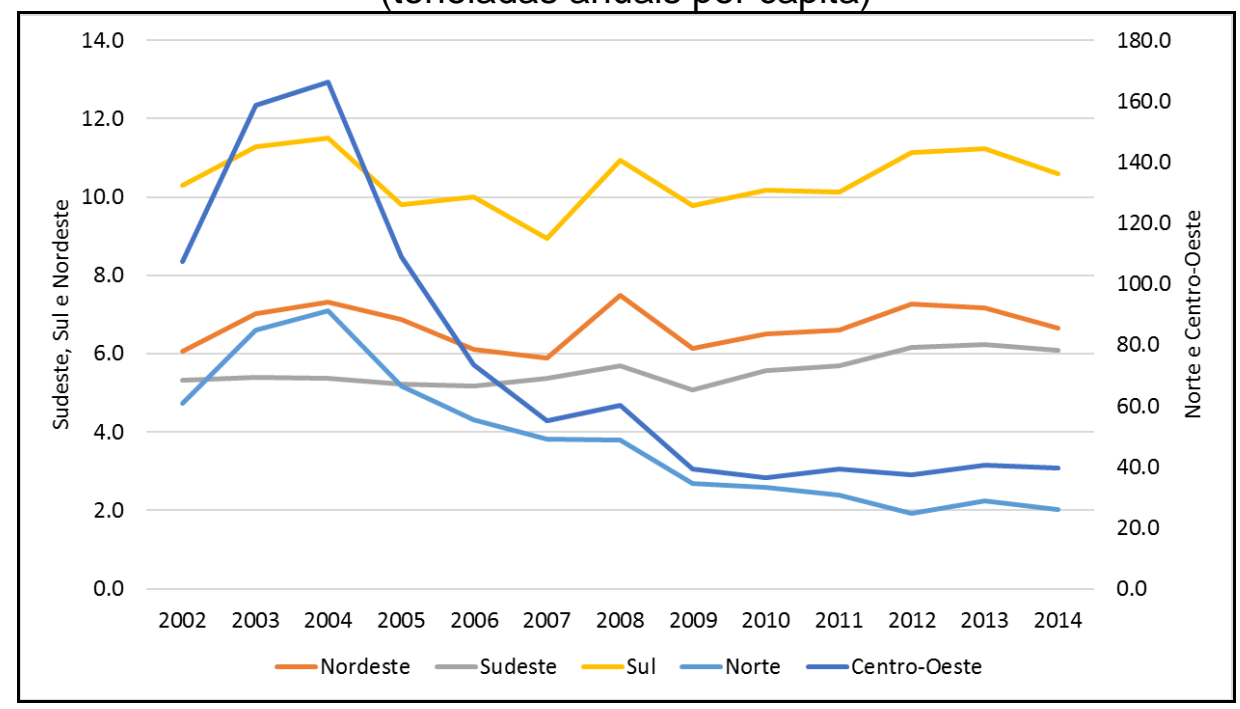

Fonte: elaborados pelos autores.

As regiões Norte e Centro-Oeste têm níveis mais elevados de emissão em função das suas características de atividade produtiva. São as regiões com maiores áreas de agronegócio, com exploração intensiva, o que está coadunado com níveis mais elevados de desmatamento - que acaba sendo captado nas estatísticas de emissão pela categoria de "mudança de uso da terra". Neste contexto, a queda mais intensa nas emissões de CO2 nestas regiões a partir de 2004 está associada ao Plano de Ação para Prevenção e Controle do Desmatamento Amazônia Legal (PPCDAm), segundo o Ministério do Meio Ambiente (MMA, 2016).

Para as demais regiões, chama a atenção a retomada do crescimento do das emissões a partir de 2009. Segundo o SEEG, particularmente a partir de 2013, isso se deve à "...subida das emissões motivada pelo aumento do desmatamento na Amazônia e pelo aumento do uso de combustíveis fósseis na matriz energética" (SEEG, 2018, p. 11)

Estes resultados, assim apresentados, reascendem algum alerta, especialmente quando observamos os compromissos assumidos junto à comunidade internacional de reduzir as emissões até 2030 no Acordo de Paris. E isto é especialmente preocupante quando se considera 0 aumento do desmatamento nos últimos períodos e os novos padrões de manejo das terras, além do uso de combustíveis fósseis.

Os níveis de bem-estar observados no Brasil melhoraram ao longo destes primeiros anos do século 21 - pelo menos no que diz respeito às variáveis analisadas neste trabalho. Na Figura 2 estão plotadas a evolução da expectativa de 
vida média das regiões brasileiras e o percentual de pobreza médio. Como se observa, a expectativa de vida ao nascer na região Sul já ultrapassa os 77 anos. E ainda que bem abaixo do Sul e Sudeste, as condições para as regiões Norte e Nordeste também melhoraram. A mesma tendência é observada para o caso da pobreza, que mostrou forte redução no período analisado. Neste caso, a queda nos índices das regiões Norte e Nordeste são especialmente significativos.

Figura 02 - Expectativa de vida ao nascer média e percentual de pobres médio por região do Brasil

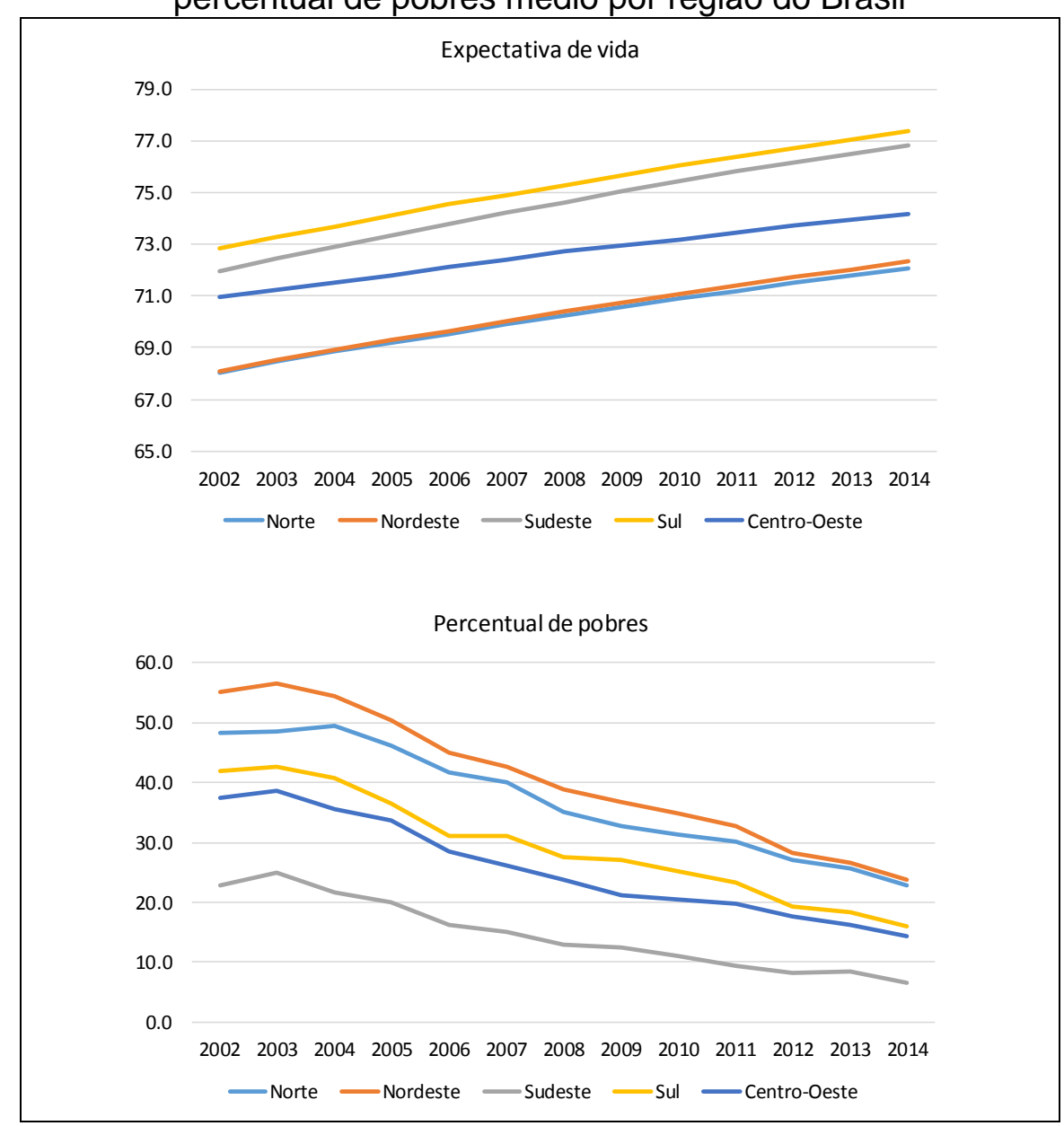

Fonte: elaborados pelos autores.

Portanto, percebe-se, de modo geral, redução e posterior estabilidade nas médias de emissão e melhoria constante nas variáveis de qualidade de vida. $A$ associação entre estas duas dimensões, por Estado, resulta na variável chave deste trabalho: intensidade ecológica do bem-estar humano (EIWB). Na Figura 03 está esboçado o comportamento das duas variáveis de EIWB propostas neste estudo, ao longo da série de tempo por região do Brasil. 
Figura 03 - Intensidade ecológica do bem-estar humano (IEWB)

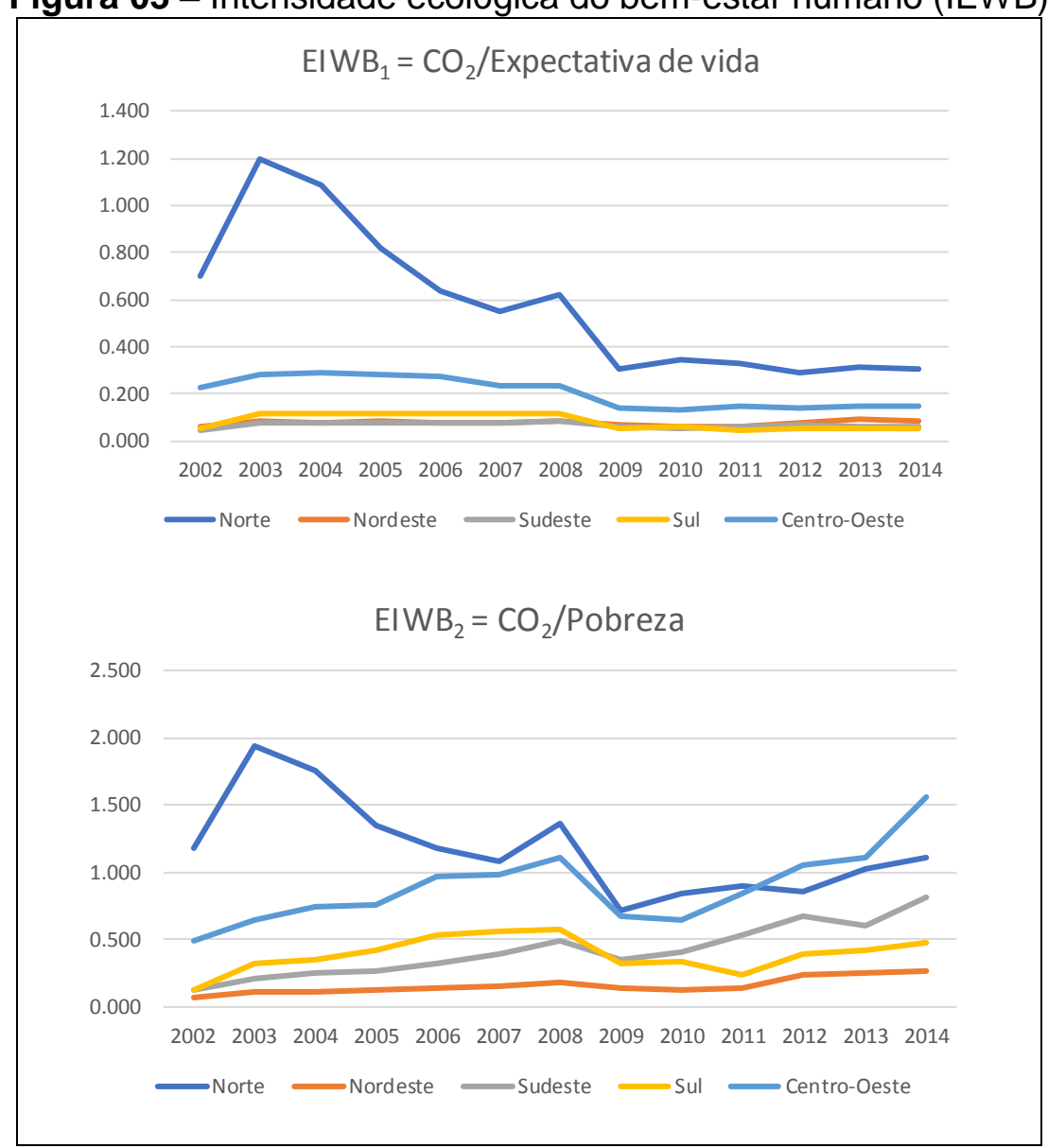

Fonte: elaborados pelos autores.

As reduções mais marcadas se observam na EIWB1, associada à expectativa de vida. Quando esta variável de bem-estar é considerada, fica evidente que ao longo da série estudada, sobretudo para as regiões Norte e Centro-Oeste, houve diminuição na pressão ambiental para cada fração adicional de expectativa de vida. O mesmo padrão não se observada com relação à pobreza, na EIWB2. A partir de 2009 há uma inversão de comportamento, sendo resultado de uma retomada, ainda que não intensa, das emissões per capita e de uma desaceleração na queda dos níveis de pobreza.

A questão deste trabalho é verificar a existência de associação entre a dinâmica da variável EIWB e o crescimento econômico. Estamos buscando avaliar qual a importância da dinâmica econômica na relação entre o que se exige do meio ambiente e os resultados em termos de bem-estar humano. Assim, o modelo econométrico proposto vai avaliar o quanto o PIB per capita é significativo para explicar os movimentos da EIWB. A Figura 04 apresenta a evolução do PIB per capita para as regiões brasileiras. 
Figura 04 - Produto Interno Bruto (PIB) per capita

das regiões do Brasil - $R \$ 1.000,00$ de 2014

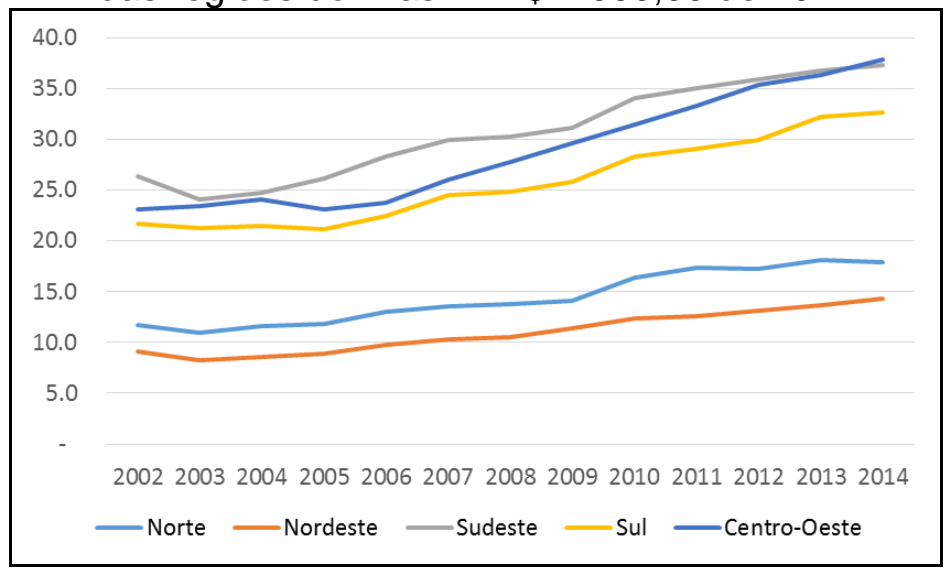

Fonte: elaborados pelos autores.

Os resultados, assim, da relação entre dinâmica econômica e EIWB estão representados nos coeficientes estimados nos modelos. A Tabela 2 traz dois modelos estimados para o Brasil como um todo pelo método descrito na metodologia, um para cada variável dependente proposta. É possível notar que a composição do PIB é significativa para explicar o comportamento da pressão ambiental nas duas variáveis, sendo que a indústria e serviços têm impacto negativo - quanto maior a participação destes setores, menor a pressão ambiental para geração de bem-estar.

O que nos importa avaliar nestes modelos estimados, de modo especial, são os coeficientes que representam o impacto do PIB per capita sobre o EIWB para cada ano da série estudada, ou seja as interações $\left[\beta_{i t}\right.$ PIB per capita $a_{i t}$ ano $t$. Metade dos coeficientes anuais para EIWB1 não são significativos a 10\%, indicado que para estes períodos o crescimento econômico não gerou impacto particularmente significativo sobre a pressão ambiental do bem-estar. Já para o caso da EIWB2, apenas o interregno 2003-2005 não foi estatisticamente significativo.

Tabela 02 - Modelos estimados para Brasil

\begin{tabular}{l|ccc|ccc}
\hline & \multicolumn{3}{|c|}{ EIWB $_{1}$} & \multicolumn{3}{c}{ EIWB $_{2}$} \\
\cline { 2 - 7 } & Coeficiente & Erro-padrão & p-value & Coeficiente & Erro-padrão & p-value \\
\hline Ln (\%PIB agro) & 0.525 & 0.117 & 0.000 & 0.454 & 0.124 & 0.000 \\
Ln (\%PIB ind) & -0.826 & 0.231 & 0.000 & -0.605 & 0.250 & 0.016 \\
Ln (\%PIB serv) & -1.683 & 0.620 & 0.007 & -1.429 & 0.658 & 0.030 \\
Ln (Gini) & -0.337 & 0.545 & 0.536 & -1.021 & 0.538 & 0.058 \\
LN (Pop ativa) & -0.823 & 0.266 & 0.002 & -0.355 & 0.351 & 0.311 \\
Ln PIB*ano & & & & & & \\
2002 (ref.) & 0.823 & 0.192 & 0.000 & 1.234 & 0.206 & 0.000 \\
2003 & 0.086 & 0.048 & 0.073 & 0.031 & 0.054 & 0.567 \\
2004 & -0.083 & 0.073 & 0.256 & -0.088 & 0.081 & 0.277
\end{tabular}




\begin{tabular}{l|ccc|ccc}
2005 & 0.067 & 0.088 & 0.449 & 0.099 & 0.099 & 0.316 \\
2006 & 0.147 & 0.078 & 0.059 & 0.282 & 0.090 & 0.002 \\
2007 & 0.125 & 0.086 & 0.145 & 0.231 & 0.098 & 0.018 \\
2008 & 0.210 & 0.108 & 0.052 & 0.339 & 0.125 & 0.006 \\
2009 & 0.317 & 0.110 & 0.004 & 0.483 & 0.126 & 0.000 \\
2010 & 0.118 & 0.117 & 0.316 & 0.346 & 0.142 & 0.015 \\
2011 & 0.163 & 0.129 & 0.208 & 0.444 & 0.153 & 0.004 \\
2012 & 0.084 & 0.133 & 0.530 & 0.487 & 0.157 & 0.002 \\
2013 & 0.196 & 0.132 & 0.137 & 0.539 & 0.150 & 0.000 \\
2014 & 0.290 & 0.145 & 0.045 & 0.755 & 0.167 & 0.000 \\
Constante & -2.258 & 2.084 & 0.279 & -7.372 & 2.309 & 0.001 \\
& & & & & & \\
$\mathrm{R}^{2}$ & & 0.4850 & & & 0.4490 & \\
rho* & & 0.7797 & & & 0.7972 & \\
Observações & & 338 & & & 338 & \\
\hline
\end{tabular}

Fonte: elaborado pelos autores.

* rho: parâmetro de autocorrelação.

** Os coeficientes angulares para os anos foram omitidos da tabela.

Tendo em mente que os coeficientes podem ser lidos como elasticidades, veja na Figura 05 o comportamento ao longo do tempo para o Brasil para as duas EIWB estimadas, calculados conforme descrito na metodologia - somando-se o coeficiente do PIB per capita (que funciona como referência para 2002) com o coeficiente de cada interação.

Figura 05 - Coeficientes estimados para PIB per capita, Brasil

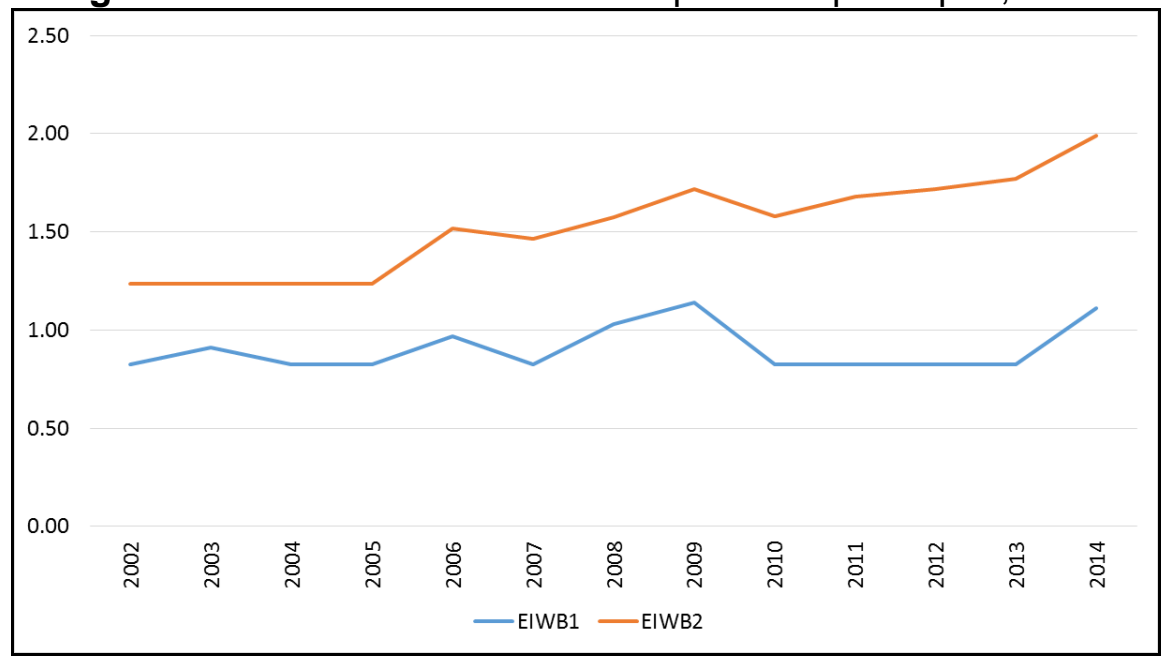

Fonte: elaborados pelos autores.

Do ponto de vista de tendência no período estudado, para ambas as variáveis dependentes, observa-se uma elevação da reação da pressão ambiental ao aumento do PIB per capita. Os números são relativamente altos, mas devem ser analisados com parcimônia, pois são reflexo de a variável dependente ser uma razão de variáveis com médias e variâncias bastante distintas. De qualquer modo, a 
mensagem é a de que o crescimento econômico tem estado associado ao aumento da pressão ambiental na geração de bem-estar ao longo os últimos anos no Brasil.

Estes resultados estão em linha com os achados de Jorgenson e Givens (2015). Estes autores demonstraram que de 1990 até 2008 os países da América Latina (fora da OCDE) tem demonstrado crescimento do impacto do crescimento do PIB per capita sobre a emissão de dióxido de carbono a partir do consumo. Se todas as 69 nações utilizadas no estudo são consideradas ao mesmo tempo, este aumento está especialmente concentrado a partir dos anos 2000.

Da mesma forma, também aponta na mesma direção de Jorgenson (2014), que indica crescimento da elasticidade do PIB per capita em relação à intensidade de carbono do bem-estar entre 1970 e 2010. Ambos os estudos utilizam como variável de bem-estar a expectativa de vida.

A diferença central do presente trabalho, e também sua contribuição principal, é que as estimativas são para o Brasil, utilizando dados estaduais. E os resultados descritivos apresentados previamente sugerem que existem diferenças consideráveis entre as regiões do país. Então, estimamos diferentes modelos para testar o comportamento das elasticidades regionais. As Tabelas 3 e 4 trazem os coeficientes estimados para as variáveis dependentes EIWB1 e EIWB2, respectivamente. 
MATTOS, TOIGO, SOUZA

Tabela 03 - Coeficientes estimados por região, variável dependente EIWB 1

\begin{tabular}{|c|c|c|c|c|c|c|c|c|c|c|c|c|c|c|c|}
\hline & \multicolumn{3}{|c|}{ Norte } & \multicolumn{3}{|c|}{ Nordeste } & \multicolumn{3}{|c|}{ Sudeste } & \multicolumn{3}{|c|}{ Sul } & \multicolumn{3}{|c|}{ Centro-Oeste } \\
\hline & Coeficiente & $\begin{array}{c}\text { Erro- } \\
\text { padrão }\end{array}$ & $p$-value & Coeficiente & $\begin{array}{c}\text { Erro- } \\
\text { padrão }\end{array}$ & $p$-value & Coeficiente & $\begin{array}{c}\text { Erro- } \\
\text { padrão }\end{array}$ & $\begin{array}{c}p- \\
\text { value }\end{array}$ & Coeficiente & $\begin{array}{c}\text { Erro- } \\
\text { padrão }\end{array}$ & $p$-value & Coeficiente & $\begin{array}{c}\text { Erro- } \\
\text { padrão }\end{array}$ & p-value \\
\hline Ln (\%PIB agro) & 0.804 & 0.155 & 0.000 & 0.506 & 0.159 & 0.001 & 0.146 & 0.072 & 0.043 & -1.449 & 0.482 & 0.003 & 8.553 & 4.719 & 0.070 \\
\hline Ln (\%PIB ind) & -0.137 & 0.176 & 0.436 & 0.988 & 0.465 & 0.034 & 0.163 & 0.611 & 0.790 & -5.693 & 1.744 & 0.001 & 9.882 & 6.688 & 0.139 \\
\hline Ln (\%PIB serv) & -1.277 & 1.225 & 0.297 & 1.372 & 0.748 & 0.067 & -0.788 & 0.807 & 0.329 & -6.039 & 2.863 & 0.035 & 20.022 & 11.010 & 0.069 \\
\hline Ln (Gini) & -0.887 & 1.044 & 0.395 & -0.178 & 0.805 & 0.825 & 0.315 & 0.973 & 0.746 & -0.394 & 1.858 & 0.832 & 2.639 & 5.184 & 0.611 \\
\hline LN (Pop ativa) & -3.797 & 0.929 & 0.000 & 0.872 & 1.622 & 0.591 & 4.674 & 11.118 & 0.674 & -15.993 & 16.091 & 0.320 & -2.009 & 6.370 & 0.753 \\
\hline \multicolumn{16}{|l|}{ Ln PIB*ano } \\
\hline 2002 (ref.) & -2.927 & 0.753 & 0.000 & -1.374 & 0.460 & 0.003 & -1.084 & 0.316 & 0.001 & 2.596 & 1.604 & 0.105 & -1.502 & 5.589 & 0.788 \\
\hline 2003 & 2.315 & 0.328 & 0.000 & -0.353 & 0.144 & 0.014 & -0.150 & 0.042 & 0.000 & 5.676 & 5.226 & 0.277 & -0.268 & 1.142 & 0.814 \\
\hline 2004 & 1.896 & 0.429 & 0.000 & -0.074 & 0.174 & 0.672 & -0.150 & 0.061 & 0.013 & 6.307 & 1.612 & 0.000 & 0.869 & 1.219 & 0.476 \\
\hline 2005 & 1.842 & 0.497 & 0.000 & 0.126 & 0.208 & 0.544 & -0.071 & 0.084 & 0.395 & 1.754 & 0.956 & 0.066 & 2.757 & 1.155 & 0.017 \\
\hline 2006 & 1.966 & 0.499 & 0.000 & 0.842 & 0.309 & 0.006 & -0.133 & 0.074 & 0.072 & 2.106 & 1.642 & 0.200 & 3.183 & 1.572 & 0.043 \\
\hline 2007 & 2.005 & 0.534 & 0.000 & 0.529 & 0.237 & 0.025 & -0.036 & 0.066 & 0.586 & 5.200 & 2.665 & 0.051 & 2.569 & 0.756 & 0.001 \\
\hline 2008 & 2.211 & 0.559 & 0.000 & 0.133 & 0.296 & 0.653 & -0.039 & 0.089 & 0.666 & 2.455 & 2.635 & 0.352 & 2.349 & 0.901 & 0.009 \\
\hline 2009 & 1.517 & 0.564 & 0.007 & 0.131 & 0.295 & 0.655 & 0.140 & 0.052 & 0.008 & 2.209 & 2.666 & 0.407 & 1.395 & 0.966 & 0.149 \\
\hline 2010 & 2.673 & 0.686 & 0.000 & 0.423 & 0.291 & 0.146 & 0.227 & 0.093 & 0.015 & 1.261 & 2.434 & 0.604 & 2.578 & 1.271 & 0.042 \\
\hline 2011 & 1.872 & 0.693 & 0.007 & 0.746 & 0.364 & 0.040 & 0.357 & 0.131 & 0.006 & 0.155 & 2.723 & 0.955 & 3.455 & 1.404 & 0.014 \\
\hline 2012 & 1.810 & 0.763 & 0.018 & 0.058 & 0.296 & 0.844 & 0.156 & 0.119 & 0.189 & 1.331 & 1.980 & 0.502 & 2.690 & 1.005 & 0.007 \\
\hline 2013 & 2.510 & 0.830 & 0.002 & 0.117 & 0.322 & 0.715 & 0.168 & 0.119 & 0.160 & 3.027 & 3.469 & 0.383 & 2.490 & 1.487 & 0.094 \\
\hline 2014 & 2.049 & 0.818 & 0.012 & -0.011 & 0.385 & 0.977 & 0.138 & 0.116 & 0.234 & 3.079 & 2.188 & 0.159 & 4.970 & 2.448 & 0.042 \\
\hline Constante & 28.136 & 9.990 & 0.005 & 0.691 & 3.861 & 0.858 & 12.144 & 7.607 & 0.110 & 13.401 & 27.056 & 0.620 & -113.381 & 69.894 & 0.105 \\
\hline $\mathrm{R}_{2}$ & & 0.6695 & & & 0.7449 & & & 0.9817 & & & 0.9219 & & & 0.7498 & \\
\hline rho & & 0.5858 & & & 0.7629 & & & 0.6175 & & & -0.0591 & & & 0.2374 & \\
\hline Observações & & 91 & & & 117 & & & 52 & & & 39 & & & 39 & \\
\hline
\end{tabular}

Fonte: elaborado pelos autores. * Os coeficientes angulares para os anos foram omitidos da tabela. 
MATTOS, TOIGO, SOUZA

Tabela 04 - Coeficientes estimados por região, variável dependente EIWB 2

\begin{tabular}{|c|c|c|c|c|c|c|c|c|c|c|c|c|c|c|c|}
\hline & \multirow{2}{*}{\multicolumn{3}{|c|}{ Norte }} & \multirow{2}{*}{\multicolumn{3}{|c|}{ Nordeste }} & \multirow{2}{*}{\multicolumn{3}{|c|}{ Sudeste }} & \multirow{2}{*}{\multicolumn{3}{|c|}{ Sul }} & \multirow{2}{*}{\multicolumn{3}{|c|}{ Centro-Oeste }} \\
\hline & & & & & & & & & & & & & & & \\
\hline & Coeficiente & $\begin{array}{c}\text { Erro- } \\
\text { padrão }\end{array}$ & $p$-value & Coeficiente & $\begin{array}{c}\text { Erro- } \\
\text { padrão }\end{array}$ & $\begin{array}{c}\mathrm{p}- \\
\text { value }\end{array}$ & Coeficiente & $\begin{array}{c}\text { Erro- } \\
\text { padrão }\end{array}$ & $p$-value & Coeficiente & $\begin{array}{c}\text { Erro- } \\
\text { padrão }\end{array}$ & p-value & Coeficiente & $\begin{array}{c}\text { Erro- } \\
\text { padrão }\end{array}$ & $p$-value \\
\hline Ln (\%PIB agro) & 0.947 & 0.177 & 0.000 & 0.353 & 0.181 & 0.051 & 0.239 & 0.108 & 0.027 & -4.628 & 0.715 & 0.000 & 2.201 & 3.037 & 0.469 \\
\hline Ln (\%PIB ind) & -0.143 & 0.229 & 0.532 & 0.743 & 0.519 & 0.152 & -1.059 & 0.828 & 0.201 & -15.375 & 2.657 & 0.000 & 8.077 & 4.318 & 0.061 \\
\hline Ln (\%PIB serv) & -1.433 & 1.427 & 0.315 & 0.642 & 0.888 & 0.470 & -2.297 & 1.078 & 0.033 & -23.442 & 4.281 & 0.000 & 6.914 & 7.132 & 0.332 \\
\hline Ln (Gini) & -1.558 & 1.205 & 0.196 & -0.701 & 0.901 & 0.437 & -2.003 & 1.470 & 0.173 & 0.490 & 2.759 & 0.859 & 4.043 & 3.321 & 0.223 \\
\hline LN (Pop ativa) & -4.296 & 1.141 & 0.000 & 3.534 & 1.885 & 0.061 & 10.893 & 15.729 & 0.489 & 61.063 & 24.650 & 0.013 & -6.898 & 4.094 & 0.092 \\
\hline \multicolumn{16}{|l|}{ Ln PIB*ano } \\
\hline 2002 (ref.) & -2.981 & 0.856 & 0.000 & -0.465 & 0.487 & 0.340 & -0.512 & 0.471 & 0.277 & 5.789 & 2.540 & 0.023 & 2.395 & 3.572 & 0.502 \\
\hline 2003 & 2.551 & 0.370 & 0.000 & -0.330 & 0.122 & 0.007 & 0.015 & 0.068 & 0.828 & -7.308 & 8.276 & 0.377 & -0.307 & 0.737 & 0.677 \\
\hline 2004 & 2.046 & 0.489 & 0.000 & -0.075 & 0.168 & 0.654 & -0.273 & 0.101 & 0.007 & 4.755 & 2.880 & 0.099 & 1.356 & 0.805 & 0.092 \\
\hline 2005 & 2.113 & 0.569 & 0.000 & 0.225 & 0.197 & 0.254 & 0.129 & 0.122 & 0.292 & 0.777 & 1.761 & 0.659 & 0.775 & 0.749 & 0.301 \\
\hline 2006 & 2.151 & 0.573 & 0.000 & 1.090 & 0.342 & 0.001 & 0.089 & 0.099 & 0.371 & 0.328 & 2.611 & 0.900 & 1.223 & 0.998 & 0.220 \\
\hline 2007 & 2.050 & 0.622 & 0.001 & 0.595 & 0.231 & 0.010 & 0.071 & 0.083 & 0.391 & -0.556 & 3.981 & 0.889 & 0.107 & 0.484 & 0.825 \\
\hline 2008 & 2.353 & 0.660 & 0.000 & 0.364 & 0.316 & 0.249 & -0.134 & 0.117 & 0.251 & -4.000 & 3.946 & 0.311 & 0.721 & 0.599 & 0.229 \\
\hline 2009 & 1.931 & 0.659 & 0.003 & 0.454 & 0.306 & 0.138 & -0.004 & 0.074 & 0.955 & -2.817 & 4.114 & 0.494 & 0.629 & 0.635 & 0.322 \\
\hline 2010 & 3.134 & 0.796 & 0.000 & 0.807 & 0.291 & 0.006 & -0.119 & 0.123 & 0.335 & -3.284 & 3.715 & 0.377 & 2.992 & 0.819 & 0.000 \\
\hline 2011 & 2.213 & 0.803 & 0.006 & 1.389 & 0.357 & 0.000 & -0.247 & 0.174 & 0.157 & -6.328 & 4.067 & 0.120 & 3.780 & 0.892 & 0.000 \\
\hline 2012 & 2.044 & 0.896 & 0.023 & 0.781 & 0.329 & 0.018 & -0.110 & 0.154 & 0.478 & -0.331 & 3.012 & 0.912 & 3.836 & 0.628 & 0.000 \\
\hline 2013 & 3.132 & 0.953 & 0.001 & 0.915 & 0.342 & 0.007 & -0.043 & 0.151 & 0.775 & 0.961 & 5.143 & 0.852 & 4.137 & 0.949 & 0.000 \\
\hline 2014 & 2.970 & 0.941 & 0.002 & 1.061 & 0.411 & 0.010 & -0.851 & 0.151 & 0.000 & 4.287 & 3.354 & 0.201 & 6.132 & 1.566 & 0.000 \\
\hline Constante & 28.719 & 11.305 & 0.011 & -2.887 & 4.361 & 0.508 & 17.914 & 10.288 & 0.082 & 111.161 & 42.370 & 0.009 & -77.591 & 45.271 & 0.087 \\
\hline $\mathrm{R}_{2}$ & & 0.6066 & & & 0.7129 & & & 0.9782 & & & 0.9921 & & & 0.7590 & \\
\hline rho & & 0.6188 & & & 0.8006 & & & 0.4193 & & & -0.1637 & & & 0.2555 & \\
\hline Observações & & 91 & & & 117 & & & 52 & & & 39 & & & 39 & \\
\hline
\end{tabular}

Fonte: elaborado pelos autores. * Os coeficientes angulares para os anos foram omitidos da tabela. 
Os resultados regionais indicam que para as regiões Norte e Centro-Oeste os coeficientes são significativos para quase todos os anos da série estudada, quando a variável é a EIWB1. Quando a variável em tela é a EIWB2, as regiões Norte e Nordeste se destacam. Para as regiões Sul e Sudeste a relação entre crescimento econômico e pressão ambiental do bem-estar não é clara, o que é demonstrado através da existência de poucos coeficientes significativos - mesmo para as variáveis de controle.

Com relação a composição do PIB, estritamente, podemos notar que alguns setores são significativos para explicar o comportamento da pressão ambiental em algumas regiões. O crescimento do setor agropecuário incide, com até $90 \%$ de confiança, em um aumento da pressão ambiental do bem-estar nas regiões Norte, Nordeste e Sudeste, independentemente da variável ser representada por EIWB1 ou EIWB2. Observamos um resultado oposto para a região Sul, cujo setor primário além de impactar negativamente, expressa uma magnitude superior. Deste resultado temos que a agropecuária não só está associada com um aumento do bem-estar e uma redução da emissão de dióxido de carbono, o que muito provavelmente se justifica pelas características e dinâmicas do setor na região sul, como o impacto negativo do crescimento econômico na pressão ambiental do bem-estar é demasiado forte.

Para a região sul, a mesma interpretação se aplica quando observamos os coeficientes dos setores industrial e de serviços, bem como suas significâncias. Os demais resultados apenas indicam que o efeito do PIB no bem estar e na pressão ambiental nas outras regiões (sozinhas) não pode ser interpretado para além de uma relação aleatória, considerando o período analisado e as variáveis de controle inseridas.

Notamos, também, que as regiões Norte e Centro-Oeste respondem por aproximadamente $85 \%$ das emissões de $\mathrm{CO} 2$ equivalente GWP AR5 do país na série considerada. Associe isso ao fato de que sua produção beneficia o país todo através da cadeia do agronegócio e da extração de recursos naturais e teremos a conclusão de que a pressão ambiental daqueles estados não é toda convertida em bem-estar local, mas sim compartilhada, em alguma medida, por todas as unidades da federação - a recíproca sendo verdadeira para as demais regiões.

Desse modo, quando avaliamos as elasticidades PIB vs. EIWB, a regionalização não agrega outras conclusões que não contra factuais. Ou seja, de que os efeitos multiplicadores da atividade regional sobre o bem-estar da nação 
como um todo são suficientemente grandes para justificar que, ainda que tenhamos os estados como unidade de análise, o foco deve ser, de fato, o país.

Por fim, cabe ressaltar que no caso de países como o Brasil, cuja base produtiva é predominantemente assentada em recursos naturais e a cadeia primária, e onde há forte dependência dos serviços ecossistêmicos, os resultados encontrados atendem ao senso comum estabelecido em termos de avaliação de impacto ambiental, uma vez que o bem-estar ainda está fortemente associado ao crescimento econômico.

\section{Considerações Finais}

Este trabalho procurou explorar a relação entre o crescimento econômico e a pressão ambiental do bem-estar humano no Brasil. Ainda que exista alguma literatura sobre o tema, a contribuição deste trabalho é discutir empiricamente este tema para um país isoladamente, e buscando uma abordagem regional.

O Brasil é um país onde as emissões de gases efeito estufa passaram por um processo de retração no início dos anos 2000, mas que tem apresentado alguma reversão desta tendência nos últimos anos. De outro lado, a geração de bem-estar, compreendida aqui como a expectativa de vida e nível de pobreza, avançou consideravelmente ao longo de todo o período estudado - ainda que com leve perda de fôlego nos últimos anos da série.

Os resultados encontrados indicam que o crescimento econômico brasileiro vem impondo custos crescentes ao meio ambiente. Ou seja, a atividade econômica tem aumentado o seu impacto sobre a pressão ambiental exercida para cada incremento no bem-estar. Este resultado, como discutido, está alinhado com os achados da literatura para países em desenvolvimento.

Algumas limitações deste trabalho precisam ser consideradas, no entanto. A principal delas, cremos, é que a regionalização se mostrou uma estratégia delicada, em função do impacto nacional das atividades econômicas, o que dificulta isolar o efeito local do crescimento econômico e da pressão ambiental. Uma estratégia seria lidar com variáveis de efeito mais local, tanto na dimensão ambiental quanto econômica. 


\section{REFERÊNCIAS}

ARRAES, Ronaldo A., DINIZ, Marcelo B., DINIZ, Márcia J. Curva ambiental de Kuznets e desenvolvimento econômico sustentável. Revista de Economia e Sociologia Rural, v. 44, n. 03, p. 525-547, 2006.

BECK, Nathaniel. KATZ, Jonathan. What to do (and not to do) with Time-Series Cross-Section Data. The American Political Science Review, v. 89, n. 3, 1999, pp. 634-647.

CARVALHO, Terciane S.; ALMEIDA, Eduardo. A hipótese da curva de Kuznets ambiental global: uma perspectiva econométrico-espacial. São Paulo: Estudos Econômicos, v. 40, n. 03, 2010, p. 587-615.

DALY, Herman. A economia do século XXI. Porto Alegre: Mercado Aberto. 1984. $120 \mathrm{p}$.

DALY, Herman, FARLEY, Joshua. Economia Ecológica: princípios e aplicações. Intituto Piaget, 2004.

DIETZ, Thomas; ROSA, Eugene A. Rethinking the environmental impacts of population, affluence and technology. Human Ecology Review, 1994, p. 277-300.

DIETZ, Thomas; ROSA, Eugene. A. YORK, Richard. Environmentally efficient wellbeing: Is there a Kuznets curve? Applied Geography, v. 32, 2012.

DINDA, S. Environmental Kuznets curve hypothesis: a survey. Ecological economics, Elsevier, v. 49, n. 4, p. 431-455, 2004.

FAN, Ying, LIU, Lan-Cui, WU, Gang, WEI, Ying-Ming. Analysing impact factors of CO2 emissions using the STIRPAT model. Environmental Impact Assessment Review, v. 26, n. 04, 2006, p. 377-395.

FOLADORI, Guillermo. Limites do desenvolvimento sustentável. Campinas: Editora UNICAMP, São Paulo: Imprensa Oficial, 2001.

GROSSMAN, Gene M.; KRUEGER, Alan B. Environmental impacts of North American free trade agreement. Cambridge: National Bureau of Economic Research, Working Paper, n. 3914, 1991.

GRUNEWALD, N. KLASEN, Stepehen, MARTINEZ-ZARZOSO, Inmaculada, MURIS, Chris. The trade-off between income inequality and carbon dioxide emissions.

Ecological Economics, Elsevier, v. 142, p. 249-256, 2017.

IPCC - Intergovernmental Panel on Climate Change. Climate Change 2014:

Synthesis Report. Contribution of Working Groups I, II and III to the Fifth Assessment Report of the Intergovernmental Panel on Climate Change [Core Writing Team, R.K. Pachauri and L.A. Meyer (eds.)]. IPCC, Geneva, 2007, 151 pp.

IPEA - INSTITUTO DE PESQUISA ECONÔMICA APLICADA. Educação, desigualdade e redução da pobreza no Brasil. Texto para Discussão, no 2447, 2019. 
JACKSON, Tim. Prosperity Without Growth. Sustainable Development Commission, United Kingdom, Macmillan, 2009, 275 p.

JORGENSON, Andrew. DIETZ, Thomas. Economic growth does not reduce the ecological intensity of human well-being. Sustainability Science, v. 10, n.1, 2015, p. 149-156.

JORGENSON, Andrew. Economic development and the carbon intensity of human well-being. Nature Climate Change, v. 04, n. 03, 2014, p. 186-189.

JORGENSON, Andrew. GIVENS, Jennifer. The Changing Effect of Economic Development on the Consumption-Based Carbon Intensity of Well-Being, 19902008. PLoS ONE, v. 10, n. 5, 2015.

KNIGHT. Kyle W. Temporal variation in the relationship between environmental demands and well-being: a panel analysis of developed and less-developed countries. Population and Environment, v. 36, n. 1, 2014, p 32-47.

MATTOS, Ely J. FILIPPI, Eduardo E. Drivers of environmental impact: A proposal for nonlinear scenario designing. Environmental Modelling \& Software, v. 62, 2014, p. 22-32.

MEADOWS, Donela H., MEADOWS, Dennis L., RANERS, Jorgen, BEHRENS, Williams W. The Limits to growth. New York: Universe Books, 1972.

MA - MILLENIUM ECOSYSTEM ASSESSMENT. Ecosystem and human wellbeing: a framework for assessment. World Resource Institute, Washington D.C.: Island Press, 2003.

MA - MILLENIUM ECOSYSTEM ASSESSMENT. Ecosystems and Human Wellbeing: Synthesis. Island Press, Washington, DC, 2005.

MMA - MINISTÉRIO DO MEIO AMBIENTE. PLANOS DE AÇÃO PARA A PREVENÇÃO E O CONTROLE DO DESMATAMENTO. Documento de base:

contexto e análises. (versão preliminar aprovada pelo GPTI), 2016. Disponível em: http://www.mma.gov.br/images/arquivo/80120/PPCDAm\%20e\%20PPCerrado\%20\%20Encarte\%20Principal\%20-\%20GPTI\%20_\%20p\%20site.pdf. Último acesso em: 27/04/2019.

PARRIS, Thomas. M. KATES, Robert W. Characterizing and measuring sustainable development. Annual Review of Environment and Resources, v. 28, 2003, p. 559586.

RAVALLION, Martin; et al. (2000). Carbon emissions and income inequality. Oxford Economic Papers, v. 52, p. 651-669.

ROMEIRO, Ademar Ribeiro. Globalização e Meio Ambiente. Campinas: IE/UNICAMP, Texto para Discussão n. 19, nov. 1999. 
SEEG - SISTEMA DE ESTIMATIVA DE EMISSÕES DE GASES DE EFEITO ESTUFA. Emissões de GEE no Brasil e suas implicações para políticas públicas e a contribuição brasileira para o Acordo de Paris. Documento de Análise 2018. 2018.

SEN, Amarty K. Desigualdade Reexaminda. Rio de Janeiro: Record, 2001.

STEINBERGER, Julia K., ROBERTS, J. Timmons, PETERS, Glen P., BAIOCCHI, Giovanni. Pathways of human development and carbon emissions embodied in trade. Nature Climate Change, v. 02, n. 2, 2012.

UNDP - UNITED NATIONS DEVELOPMENT PROGRAMME. Human Development Report 1990. New York: Oxford University Press, 1990.

TEEB - THE ECONOMICS OF ECOSYSTEMS AND BIODIVERSITY. A economia dos ecossistemas e da biodiversidade: integrando a economia da natureza. Uma síntese da abordagem, conclusões e recomendações do TEEB. PNUMA, 2010.

WACKERNAGEL, Mathis; REES, Williams E. (1996). Our ecological footprint, reducing human impact on the earth. Philadelphia: New Society Publishers, 1996.

\section{NOTAS DE AUTOR}

\section{CONTRIBUIÇÃO DE AUTORIA}

Ely José de Mattos - Concepção. Análise de dados. Elaboração do manuscrito, revisão e aprovação da versão final do trabalho

Camila Horst Toigo - Elaboração do manuscrito. Coleta de dados. Participação ativa da discussão dos resultados. Revisão e aprovação da versão final do trabalho.

Osmar Tomaz de Souza - Elaboração do manuscrito. Participação ativa da discussão dos resultados. Revisão e aprovação da versão final do trabalho.

\section{FINANCIAMENTO}

Não se aplica.

\section{CONSENTIMENTO DE USO DE IMAGEM}

Não se aplica.

APROVAÇÃO DE COMITÊ DE ÉTICA EM PESQUISA

Não se aplica.

\section{CONFLITO DE INTERESSES}

Não há conflito de interesse.

\section{LICENÇA DE USO}

Este artigo está licenciado sob a Licença Creative Commons CC-BY. Com essa licença você pode compartilhar, adaptar, criar para qualquer fim, desde que atribua a autoria da obra.

\section{HISTÓRICO}

Recebido em: 27-04-2019

Aprovado em: 28-05-2020 